\title{
COMUNIDADES ZOOPLANCTÓNICAS EN LAGOS DEL PARQUE NACION- AL TORRES DEL PAINE: UN NUEVO ENFOQUE DE ANÁLISIS DE FACTORES REGULADORES DE SU ESTRUCTURA COMUNITARIA
}

\author{
ZOOPLANKTONIC COMMUNITIES IN LAKES FROM TORRES DEL PAINE NATIONAL \\ PARK: A REASSESSMENT OF REGULATING FACTORS ON COMMUNITY STRUCTURE.
}

Patricio De los Ríos ${ }^{1}$, Doris Soto ${ }^{2} \&$ Andrés Mansilla ${ }^{3}$

\begin{abstract}
RESUMEN
El Parque Nacional Torres del Paine se caracteriza por la presencia de una serie de ecosistemas lacustres tales como grandes lagos ultraoligotróficos, lagos pequeños con peces, y lagunas pequeñas superficiales. Los estudios publicados sobre la base de información obtenida entre los años 1989 y 1991 , indican que a la riqueza de especies está indirectamente relacionada con la conductividad y directamente relacionada con la concentración de clorofila. El presente trabajo consistió en un análisis de información de algunos lagos del parque sin publicar, obtenida el año 2001, donde además se consideró concentración de carbono orgánico disuelto como un agente protector contra el incremento de la radiación ultravioleta natural reportado en las últimas dos décadas. Los resultados mostraron tendencias similares a las observadas en los primeros registros, donde se observó un rol de la concentración de clorofila y la conductividad en la riqueza de especies; a lo que se agrega ahora la concentración de carbono orgánico disuelto, la cual estaría directamente relacionada con la riqueza de especies. La máxima riqueza de especies se observa en condiciones de mesotrofía, baja conductividad y alta concentración de carbono orgánico disuelto. Estos resultados concordarían parcialmente con la aplicación de modelos nulos de asociaciones de especies que indican la existencia de factores reguladores.
\end{abstract}

Palabras clave: mesotrofía, oligotrofía, conductividad, zooplancton, radiación ultravioleta.

The Torres del Paine National Park is characterized by the presence of numerous water bodies, such as large oligotrophic lakes, small lakes with fishes, and small shallow ponds. The published studies, based on data obtained between 1989 and 1991, indicates that species richness is inversely related with the conductivity and directly related with chlorophyll concentration. The present study consisted of an analysis from unpublished data obtained during 2001, where it was also considered the dissolved organic carbon concentration as a potential protector against natural ultraviolet radiation penetration, reported during the last two decades. The results were similar to the first studies on the role of chlorophyll concentration and conductivity as regulators of species richness, and in the present study was denoted that the species richness was directly related with dissolved organic carbon concentration. The maximum species richness was observed in mesotrophic status, low conductivity and high dissolved organic carbon concentration. These results agree partially with co-occurrence null model analysis.

Key words: mesotrophy, oligotrophy, conductivity, zooplankton, dissolved organic carbon.

\footnotetext{
${ }^{1}$ Laboratorio de Ecología Aplicada y Biodiversidad, Escuela de Ciencias Ambientales, Facultad de Recursos Naturales, Universidad Católica de Temuco, Casilla 15-D, Temuco, Chile. prios@uct.cl

${ }^{2}$ Senior Fisheries Officer Inland Water Resources and Aquaculture Service (FIRI), Fisheries Department, FAO of UN, Via delle Terme di Caracalla, I-00100, Roma, Italia.

${ }^{3}$ Facultad de Ciencias, Universidad de Magallanes, Avenida Bulnes 01855, Punta Arenas, Chile.
} 


\section{INTRODUCCIÓN}

El Parque Nacional Torres del Paine (PNTP) se caracteriza por la presencia de numerosos lagos y lagunas, los que podrían considerarse en tres grupos, lagos grandes oligotróficos con poblaciones de salmónidos y galáxidos, lagos chicos mesotróficos con poblaciones de galáxidos, y lagunas con condiciones tróficas variables poco profundas sin peces (Soto et al. 1994). Esta heterogeneidad de ambientes acuáticos influye sobre los ensambles zooplanctónicos, ya que en las primeras observaciones basadas en datos obtenidos entre los años 1989 a 1991, se encontró que la conductividad y el estatus trófico influyeron, encontrándose alto número de especies en condiciones de mesotrofía y conductividad moderada a baja (Soto \& De los Ríos 2006, De los Ríos \& Soto 2009).

Por otro lado, en la actualidad, se ha observado un aumento de la penetración de la radiación ultravioleta, debido a la disminución de la capa de ozono en la Antártica (Morris et al. 1995, Díaz et al. 2006, Marinone et al. 2006). En este escenario, la radiación ultravioleta, especialmente la radiación ultravioleta $\mathrm{B}$ (UVB) puede penetrar la columna de agua en condiciones de oligotrofía y bajas concentraciones de substancias que hagan un efecto de pantalla contra la radiación ultravioleta como el carbono orgánico disuelto (Morris et al. 1995). En estas condiciones, de alta penetración de la radiación ultravioleta en la columna de agua, sólo se encontrarían las especies tolerantes a la radiación, por lo que se podría suponer que la exposición a la radiación ultravioleta y su consecuente penetración en la columna de agua puede ser un regulador importante en la estructura comunitaria zooplanctónica (Marinone et al. 2006).

En este escenario, se pretende estudiar el rol potencial de la concentración de carbono orgánico disuelto (como potencial agente protector contra la penetración de la radiación ultravioleta en la columna de agua), concentración de clorofila y conductividad como potenciales factores que regularían la riqueza de especies zooplanctónicas en lagos y lagunas del Parque Nacional Torres del Paine.

\section{MATERIAL Y MÉTODOS}

Se consideró información colectada en febrero y marzo del año 2001 en lagos y lagunas del Parque Nacional Torres del Paine, y como sitio externo, una laguna externa localizada en el cordón Arauco en las cercanías de la ciudad de Puerto Natales. Se midieron en terreno a nivel superficial, la conductividad, salinidad y temperatura utilizando un sensor YSI-30, se colectaron muestras para análisis de clorofila por medio de extracción de pigmentos con acetona, esto es por filtración de $0,5 \mathrm{~L}$ de agua mediante papel Whatman GFC, el cual se sumergió en acetona por 48 horas, para su posterior lectura por medio de espectrofotometría a 360, 345 y $330 \mathrm{~nm}$ (Wetzel \& Likens 1991), y carbono orgánico disuelto siguiendo las metodologías descritas por Morris et al. (1995), lo cual consistió en filtrar 0,5 L de agua y posterior análisis por medio de utilización de un medidor de carbono orgánico total marca Shimadzu, lo cual se realizó en la Universidad de Lehigh en Estados Unidos de América.

Las muestras de zooplancton, se colectaron siguiendo las metodologías descritas por Soto \& De los Ríos (2006), i.e., muestreos diurnos, en el caso del lago del Toro, mediante lances verticales de una malla de zooplancton de $20 \mathrm{~cm}$ de diámetro y 100 $\mu \mathrm{m}$ de tamaño de poro a una profundidad de 30 metros por medio de un bote, mientras que para los demás sitios se tomaron muestras de $50 \mathrm{~L}$ con un balde, el volumen colectado se filtró por medio de un tamiz de $100 \mu \mathrm{m}$, y el material colectado se fijó con etanol. Las muestras se identificaron según las descripciones de Araya \& Zúñiga (1985), Bayly (1992) y Reid (1985). Se consideró información de superficie, profundidad, temperatura, conductividad, salinidad, concentraciones de clorofila y carbono orgánico disuelto (como agente protector natural contra la radiación ultravioleta), y número de especies de crustáceos zooplanctónicos, a estos datos se les aplicó un análisis de componentes principales, por medio del programa Xlstat 5.0. De manera referencial, se incluyó información de radiación ultravioleta para la zona de Punta Arenas, proporcionada por la Dirección Meteorológica de Chile (Tabla 1).

A los datos de asociaciones de especies se les consideró que podrían ser aleatorios; para esto se consideró aplicar el "índice de ponderación C" (Stone \& Roberts 1990), el cual se determina en la co-ocurrencia de especies basado en manejar una matriz de presencia y ausencia de especies. Sobre la base de las metodologías de Gotelli (2000), Tondoh (2006), Tiho \& Johens (2007), la matriz de presencia y ausencia se considera a las filas donde van las especies y los sitios que corresponden a las 
columnas, y sobre esta base se analizó según las siguientes simulaciones: (a) Modelo fijo-fijo: en este algoritmo las filas y columnas originales no son alteradas, y así cada simulación aleatoria contiene el mismo número de especies de la comunidad original (columna fija) y cada especie ocurre en la misma frecuencia que la comunidad original (fila fija). En esta instancia no permite la presencia de errores tipo I, que consisten en rechazar erróneamente la hipótesis nula y es muy robusto para detectar la falta de aleatorizaciones (Gotelli 2000, Tiho \& Johens 2006, Tondoh 2007). (b) Modelo fijo-equiprobable: en esta simulación sólo la suma de las columnas está fijada, mientras que las columnas que corresponden a los sitios se consideran como equiprobables. Este modelo nulo considera todos los sitios (correspondientes a las columnas de la matriz) como igualmente disponibles para todas las especies (Tiho \& Johens 2006, Gotelli 2000). (c) Modelo fijo-proporcional: en este algoritmo la ocurrencia total de especies es mantenida como en la comunidad original, y la probabilidad que alguna especie se presente en un sitio (columna) es proporcional al total de la columna por sitio (Gotelli 2000, Tiho \& Johens 2006, Tondoh 2007). Los datos fueron analizados mediante el programa Ecosim versión 7.0 (Gotelli \& Entsminger 2009).

\section{RESULTADOS Y DISCUSIÓN}

Los resultados de la tabla 2, muestran que el lago del Toro, tiende a ser diferente debido a su oligotrofía, baja conductividad y concentración de carbono orgánico disuelto, y baja riqueza de especies, mientras que los lagos chicos y las lagunas tienden a ser mesotróficos o eutróficos, con valores relativamente moderados en cuanto a sus concentraciones carbono orgánico disuelto y al número de especies zooplanctónicas. (Tabla 2). La excepción podría ser la laguna Cisnes, que presentó alta conductividad, altas concentraciones de clorofila y carbono orgánico disuelto y bajo número de especies (Tabla 2). Estos resultados concuerdan con un primer estudio donde se observó que los lagos patagónicos profundos donde tienen baja riqueza de especies asociado a condiciones de oligotrofia (Campos 1984, Campos et al. 1994a,b; Soto \& Zúñiga 1991, Modenutti et al. 1998, Quiroz \& Drago 1999, Woelfl 2007).

Los resultados de la matriz de correlación, mostraron relaciones directas significativas entre área y profundidad, de igual modo hubo similar tendencia entre salinidad, conductividad, carbono orgánico disuelto y concentración de clorofila, mientras que sólo hubo una diferencia significativa inversa entre número de especies con profundidad (Tabla 3). Estos resultados se deberían, presumiblemente, a la alta evaporación a la que están expuestos estos ecosistemas debido a la exposición a los fuertes vientos, lo que repercute en las altas concentraciones de substancias disueltas como se ha observado en lagunas (Soto et al. 1994, Campos et al. 1996), mientras que en lagos profundos tendríamos la situación contraria (Campos et al. 1994a,b). Resultados similares han sido observados para lagos de la Patagonia de Argentina (Quirós \& Drago 1999).

Los resultados del Análisis de Componentes Principales revelaron que las variables que contribuyeron con el 83,96\%, para el primer eje la contribución fue de $54,07 \%$ y las variables importantes fueron conductividad, salinidad, clorofila "a" y concentración de carbono orgánico disuelto (Fig. 1a). Mientras que para el segundo eje, la contribución fue de un $29,89 \%$, y las variables importantes fueron superficie, profundidad máxima, y riqueza de especies zooplanctónicas (Fig. 1a). Estos resultados denotan que las condiciones tróficas son más importantes que las características morfológicas y topográficas (área, profundidad máxima,) y riqueza de especies zooplanctónicas (Figs. 1a y 1b).

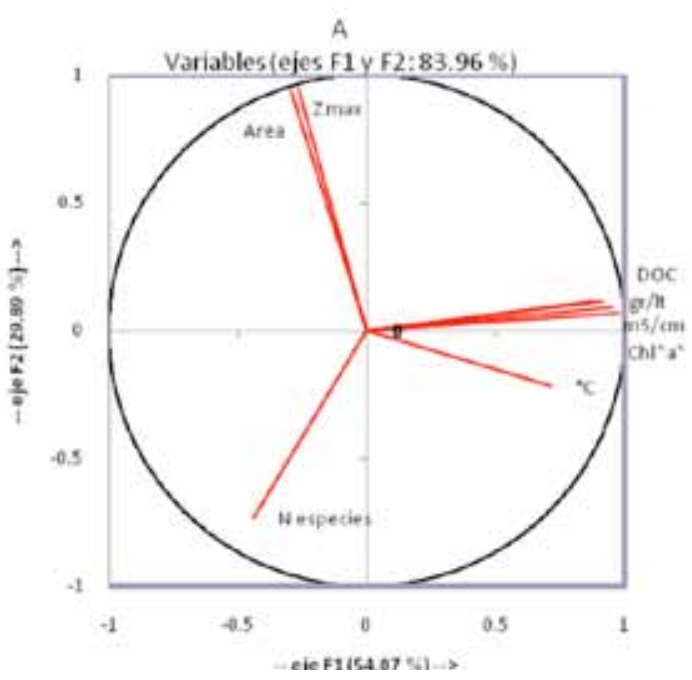

Fig. 1a. Resultados del análisis de componentes principales para los sitios estudiados. 


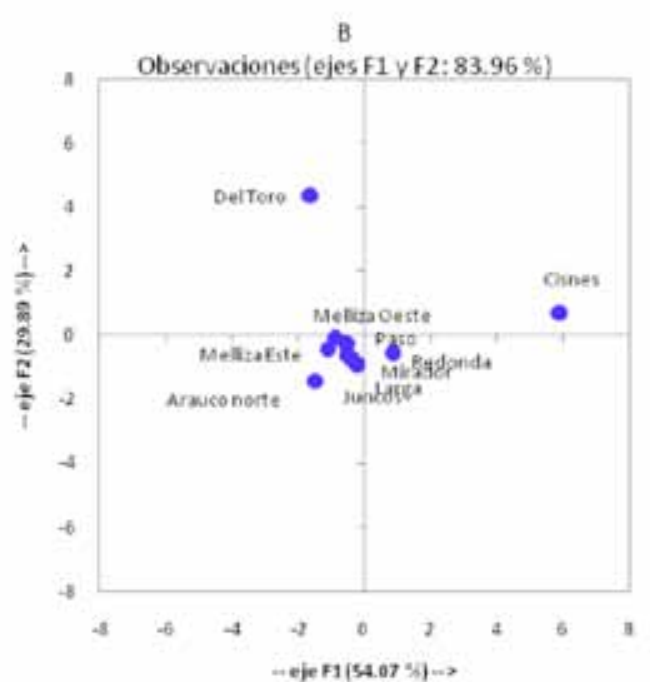

Fig. 1b. Resultados del análisis de componentes principales para los sitios estudiados.

Los resultados del Análisis de Componentes Principales, indican la existencia de dos grupos de lagos, el primero corresponde al lago del Toro que es grande, profundo y oligotrófico, y baja riqueza de especies de crustáceos zooplanctónicos (Tabla 2, Fig. 1b). El segundo grupo correspondió a lagos pequeños y lagunas poco profundas, con conductividad moderada, mesotróficos, riqueza de especies crustáceos zooplanctónicos relativamente altas (Paso, Juncos, Melliza Este y Melliza Oeste; Tabla 2; Fig. 1b). Dentro de este grupo además se incluyen las laguna Cisnes, que presentó alta conductividad, altas concentraciones de materia orgánica disuelta y clorofila "a", y baja diversidad de especies de crustáceos zooplanctónicos (Tablas 2 y 3, Fig. 1b). Sobre esta base, se podría indicar que la máxima riqueza de especies estaría en condiciones de mesotrofía, baja conductividad y alta concentración de carbono orgánico disuelto (Tabla 2, Figs. 1a y 1b). Estos resultados podrían concordar con las primeras descripciones realizadas para zooplancton de lagos de este sitio donde se encontró que la máxima diversidad presentaba mesotrofía y baja conductividad (Soto \& De los Ríos 2006, De los Ríos \& Soto 2009). Esto además, se podría integrar con las descripciones de Marinone et al. (2006) y De los Ríos et al. (2008) donde se encontró que, en condiciones de oligotrofía y alta exposición a la radiación ultravioleta, está asociada a bajas abundancias de especies zooplanctónicas.
En los primeros estudios, se propuso que el efecto de los salmónidos asilvestrados sobre la base de primeras propuestas para la zona (Soto et al. 1994, 2006, 2007; Arismendi et al. 2009) y para la Patagonia argentina (Becker et al. 2007, Pascual et al. 2007, Reissig et al. 2004, 2006) y la actividad de peces nativos, específicamente Galáxidos (Soto et al. 1994) pudo tener efecto en la regulación de las comunidades zooplanctónicas. No obstante, no se pudo confirmar ni rechazar potenciales efectos de los peces sobre el zooplancton en lagos de la Patagonia chilena (De los Ríos \& Soto 2009). Las especies registradas tienden a ser similares a las primeras descripciones (Soto \& De los Ríos 2006), donde en sitios con peces (lago del Toro, lagos chicos Melliza Este y Melliza Oeste) se encontraron especies de tamaño pequeño como por ejemplo $B$. gracilipes y $D$. pulex, mientras que en los sitios restantes sin peces, hubo especies de gran tamaño como por ejemplo Boeckella poppei y Parabroteas sarsi (Tabla 4).

Los resultados de los modelos nulos denotan la ausencia de aleatorizaciones en las asociaciones de especies en dos (modelos fijo-proporcional y fijo-equiprobable) de las tres simulaciones, mientras que el modelo fijo-fijo mostró presencia ausencia de factores reguladores (Tabla 5). Estos resultados concuerdan con los obtenidos en el PCA, que indica la presencia de factores reguladores que afectarían la riqueza de especies de crustáceos zooplanctónicos, pero los resultados aparentemente contradictorios observados en el modelo fijo-fijo se deberían a la presencia de pocas especies que están presentes en casi la totalidad de los sitios estudiados, lo cual concuerda con otras descripciones similares para lagos chilenos (De los Ríos et al. 2008, De los Ríos 2008, De los Ríos \& Roa 2010). Similares resultados fueron observados en ambientes terrestres (Ribas \& Schoereder 2002, Sanders et al. 2007). Otra posible causa es la homogeneidad de hábitats (Franca \& Araujo 2007), o interacciones con especies de características ecológicas similares (Tondoh 2006, Tiho \& Johens 2007). En un caso contrario, la presencia de competencia interespecífica y la diferenciación ecológica denota la ausencia del azar en las asociaciones de especies (Ulrich 2004, Rodríguez-Fernández et al. 2006). Por otro lado, la existencia de factores reguladores, puede deberse a segregaciones de hábitats y compartimiento de 
recursos entre las especies de la comunidad (Costa de Azevedo et al. 2006).

En resumen, es probable que a contar del año 2001 podrían existir resultados atribuibles al incremento de la penetración de la radiación ultravioleta debido al debilitamiento de la capa de ozono austral. No obstante, se requiere de más información sobre la penetración de la radiación ultravioleta en la columna de agua (Marinone et al. 2006), así como procesos de extinción y colonización de nuevas especies (Ramos-Jiliberto et al. 2009), lo cual podría ser un buen ejemplo de estudio de los efectos de cambios globales, como el incremento de la exposición a la radiación ultravioleta. Por otro lado es necesario estudiar detalladamente el rol del zooplancton en las tramas tróficas, donde por un lado tenemos el efecto de peces zooplanctívoros (Soto et al. 1994), $y$ en sitios sin peces, este rol lo tendrían las aves acuáticas como los flamencos (Campos et al. 1996).

Los resultados concluirían que la concentración de carbono orgánico disuelto, como potencial agente protector contra la penetración de la radiación ultravioleta, influiría sobre la estructuración del zooplancton junto con la concentración de clorofila y la conductividad. En este escenario, la máxima riqueza de especies sucedería bajo condiciones de mesotrofía, baja conductividad y alta concentración de carbono orgánico disuelto, lo que concordaría con los resultados del análisis de modelos nulos.

TABLA 1. Datos de radiación ultravioleta para la zona de la Patagonia durante primavera y verano del año 2001 y verano de los años 2002 a 2004 (fuente: Dirección Meteorológica de Chile). Punta Arenas (53 00' S; 70 58’ W)

\begin{tabular}{ccl}
\hline Promedio mensual & Horas de insolación & \multicolumn{1}{c}{ Mes } \\
\hline 158.28 & 7.1 & Octubre 2001 \\
235.33 & 8.6 & Diciembre 2001 \\
211.20 & 7.8 & Enero 2002 \\
168.24 & 7.5 & Febrero 2002 \\
107.94 & 5.5 & Marzo 2002 \\
207.24 & 7.3 & Diciembre 2002 \\
191.74 & 8.0 & Enero 2003 \\
147.63 & 6.0 & Febrero 2003 \\
\hline Datos promedio de UVB promedio en la zona de Punta Arenas. \\
UVB (mW/m2) & Período & Mes \\
\hline 2714.09 & $2002-2004$ & Enero \\
2232.57 & $2002-2004$ & Febrero
\end{tabular}


TABLA 2. Superficie, área, profundidad máxima, conductividad, salinidad, temperatura, concentraciones de carbono orgánico disuelto y clorofila, y número de especies reportada para sitios visitados en el presente estudio.

Cisnes Redonda Juncos Larga Paso Mirador $\begin{gathered}\text { Melliza } \\ \text { Oeste }\end{gathered} \begin{gathered}\text { Melliza } \\ \text { Este }\end{gathered}$ Del Toro $\begin{gathered}\text { Arauco } \\ \text { norte }\end{gathered}$

Latitud $\quad 51^{\circ} 01^{\prime} \mathrm{S} 51^{\circ} 01^{\prime} \mathrm{S} 51^{\circ} 01^{\prime} \mathrm{S} 51^{\circ} 01^{\prime} \mathrm{S} 51^{\circ} 02^{\prime} \mathrm{S} 51^{\circ} 03^{\prime} \mathrm{S} 51^{\circ} 03^{\prime} \mathrm{S} 51^{\circ} 03^{\prime} \mathrm{S} 51^{\circ} 12^{\prime} \mathrm{S} 51^{\circ} 50^{\prime} \mathrm{W}$ $72^{\circ} 52^{\prime} \mathrm{W} 72^{\circ} 52^{\prime} \mathrm{W} 72^{\circ} 52^{\prime} \mathrm{W} 72^{\circ} 52^{\prime} \mathrm{W} 72^{\circ} 55^{\prime} \mathrm{W} 72^{\circ} 52^{\prime} \mathrm{W} 72^{\circ} 57^{\prime} \mathrm{W} 72^{\circ} 57^{\prime} \mathrm{W} 72^{\circ} 38^{\prime} \mathrm{W} 72^{\circ} 57^{\prime} \mathrm{W}$

\begin{tabular}{|c|c|c|c|c|c|c|c|c|c|c|}
\hline Area (km2) & 0.1 & 0.1 & 0.1 & 0.1 & 0.1 & 0.1 & 0.12 & 0.13 & 196 & 0.1 \\
\hline Z máxima (m) & 1.00 & 5.00 & 5.00 & 10.00 & 5.00 & 10.00 & 25.00 & 16.00 & 300.00 & 5.00 \\
\hline $\begin{array}{l}\text { Conductividad } \\
(\mathrm{mS} / \mathrm{cm})\end{array}$ & 13200.20 & 1365.00 & 403.20 & 4330.00 & 498.00 & 593.20 & 1127.50 & 1375.00 & 80.50 & 234.10 \\
\hline Salinidad (g/l) & 10.00 & 2.00 & 0.10 & 0.20 & 0.10 & 0.10 & 0.30 & 0.20 & 0.30 & 1.00 \\
\hline $\begin{array}{l}\text { Temperatura } \\
\left({ }^{\circ} \mathrm{C}\right)\end{array}$ & 17.10 & 11.40 & 16.80 & 14.20 & 11.80 & 14.30 & 10.20 & 8.10 & 9.00 & 9.00 \\
\hline $\mathrm{COD}(\mathrm{mg} / \mathrm{l})$ & 168.50 & 9.10 & 14.12 & 15.61 & 10.83 & 3.67 & 4.8 & 7.6 & 2.5 & 15.08 \\
\hline Chl "a"( $\mu \mathrm{g} / \mathrm{l})$ & 17.90 & 6.30 & 6.70 & 15.40 & 7.40 & 8.80 & 5.8 & 7.0 & 7.6 & 5.20 \\
\hline $\mathrm{N}$ especies & 2 & 4 & 4 & 4 & 3 & 4 & 3 & 4 & 2 & 6 \\
\hline
\end{tabular}

TABLA 3. Resultados de matriz de correlación de las variables consideradas en el presente trabajo, los valores en negrita indican presencia de una correlación estadísticamente significativa $(\mathrm{P}<0.05)$.

\begin{tabular}{lccccccc}
\hline & $\mathrm{Z}$ maxima & $\mathrm{mS} / \mathrm{cm}$ & $\mathrm{gr} / \mathrm{lt}$ & ${ }^{\circ} \mathrm{C}$ & $\mathrm{COD}$ & $\mathrm{Chl}$ "a" & $\mathrm{N}$ especies \\
\hline Area & 0.997 & -0.196 & -0.129 & -0.344 & -0.158 & -0.099 & -0.479 \\
Z maxima & -0.221 & -0.163 & -0.379 & -0.191 & -0.124 & -0.481 \\
$\mathrm{mS} / \mathrm{cm}$ & & & 0.933 & 0.564 & 0.959 & 0.882 & -0.435 \\
$\mathrm{gr} / \mathrm{lt}$ & & & 0.477 & 0.979 & 0.688 & -0.409 \\
${ }^{\circ} \mathrm{C}$ & & & & & 0.555 & 0.631 & -0.216 \\
$\mathrm{COD}$ & & & & & & 0.758 & -0.424 \\
Chl "a" & & & & & & -0.410 \\
\hline
\end{tabular}


TABLA 4. Matriz de presencia y ausencia de especies reportadas para los sitios estudiados en el presente trabajo.

\begin{tabular}{|c|c|c|c|c|c|c|c|c|c|c|}
\hline & Redonda & Juncos & Cisnes & Larga & Mirador & Paso & $\begin{array}{l}\text { Arauco } \\
\text { norte }\end{array}$ & $\begin{array}{l}\text { Melliza } \\
\text { Este }\end{array}$ & $\begin{array}{l}\text { Melliza } \\
\text { Oeste }\end{array}$ & DelToro \\
\hline Alona sp & 0 & 0 & 0 & 0 & 1 & 0 & 0 & 1 & 1 & 0 \\
\hline Daphnia pulex (Scourfield, 1877) & 1 & 0 & 0 & 1 & 0 & 1 & 1 & 0 & 0 & 0 \\
\hline D. obtusa (Kurz, 1874) & 0 & 0 & 0 & 0 & 0 & 0 & 1 & 0 & 0 & 0 \\
\hline Neobosmina chilensis (Daday,1902) & 0 & 0 & 0 & 0 & 0 & 0 & 1 & 1 & 1 & 0 \\
\hline Boeckella gracilipes (Daday, 1902) & 1 & 0 & 0 & 1 & 1 & 1 & 0 & 1 & 1 & 1 \\
\hline B. meteoris (Kiefer, 1928) & 0 & 0 & 1 & 0 & 0 & 0 & 0 & 0 & 0 & 0 \\
\hline B. michaelseni (Mrázek, 1901) & 0 & 0 & 0 & 0 & 0 & 0 & 1 & 0 & 0 & 0 \\
\hline B. poppei (Mrázek, 1901) & 0 & 1 & 0 & 1 & 0 & 0 & 1 & 0 & 0 & 0 \\
\hline Parabroteas sarsi (Mrázek, 1901) & 1 & 1 & 1 & 1 & 1 & 0 & 0 & 0 & 0 & 0 \\
\hline $\begin{array}{l}\text { Mesocyclops araucanus } \\
\text { (Pilati \& Menu-Marque, 2003). }\end{array}$ & 0 & 0 & 0 & 0 & 0 & 0 & 0 & 0 & 1 & 1 \\
\hline
\end{tabular}

TABLA 5. Resultados de las simulaciones del modelo nulo de co-ocurrencia de especies (valores de "p" inferiores a 0.05 indican que las asociaciones de especies no son aleatorias).

\begin{tabular}{lcccc}
\hline Modelo & Índice observado & Índice Promedio & Efecto Estándar del Tamaño & $\mathrm{P}$ \\
\hline Fijo-Fijo & 4.022 & 3.810 & 1.670 & 0.068 \\
Fijo-Proporcional & 4.022 & 3.148 & 1.833 & 0.016 \\
Fijo-Equiprobable & 4.022 & 3.394 & 1.534 & 0.042
\end{tabular}

\section{AGRADECIMIENTOS}

Los resultados del presente trabajo en terreno fueron financiados por el proyecto IAI (Enhanced Ultraviolet B Radiation in Natural Ecosystems as an Added Perturbation Due Ozone Depletion), CONICYT (Beca de Término de Tesis Doctoral y Beca Doctoral), Universidad Austral de Chile (Proyecto DID-UACH 2001d-11) y Universidad Católica de Temuco (Proyecto DGI-UCT CDA 2007-01).

\section{LITERATURA CITADA}

Abelha, M.C.F, E. Goulart, E.A.L. Kashiwaqui \& M.R Da Silva 2006. Astyanax paranae Eigenmann, 1914 (Characiformes: Characidae) in the Alagados Reservoir, Parana, Brazil: diet composition and variation. Neotropical Ichthyology, 4: 345-356.

Arismendi, I., D. Soto, B. Penaluna, C. Jara, C. Leal \& J. León-Muñoz 2009. Aquaculture, non-native salmonid invasions and associated declines of native fishes in Northern Patagonian lakes. Freshwater Biology 54: 1154-1147.

Becker, L.A., M.A. Pascual \& N.G. Basso 2006. Colonization of the southern Patagonia Ocean by exotic Chinook salmon. Conservation Biology 21: 1347-1352.

Campos, H. 1984. Limnological studies of Araucanian lakes (Chile) Verhandlungen International Vereinüng fur Angewaldte und Applied Limnolologie 22:1319-1327.

Campos, H., D. Soto, W. Steffen, G. Agüero, O. Parra \& L. Zúñiga 1996. Limnological studies of Amarga Lagoon, Chile: a saline lake in Patagonian South America. International Journal of Salt Lake Research 4:301-314.

Campos, H., D. Soto, W. Steffen, G. Agüero, O. Parra \& L. Zúñiga 1994a. Limnological studies of Lake del Toro (Chile) morphometry, physics, chemistry and plankton. Archiv für Hydrobiologie Supplement 99: 199-215.

Campos, H., D. Soto, W. Steffen, G. Agüero, O. Parra \& L Zúñiga 1994b. Limnological stu- 
dies of Lake Sarmiento (Chile): A subsaline lake from Chilean Patagonian. Archiv für Hydrobiologie Supplement 99: 217-234.

Costa de Azevedo, M.C., F.G. Araujo, A.L. Machado \& M. de Araujo Silva 2006. Cooccurrence of demersal fishes in a tropical bay in southeastern Brazil: A null model analysis. Estuarine Coastal and Shelf Science. 66: 315-322.

De los Ríos, P., N. Rivera \& M. Galindo 2008. The use of null models to explain crustacean zooplancton associations in shallow water bodies of the Magellan region, Chile. Crustaceana 81: 1219-1128

De los Ríos, P., P. Acevedo, R. Rivera \& G. Roa 2008c. Comunidades de crustáceos litorales de humedales del norte de la Patagonia chilena $\left(38^{\circ} \mathrm{S}\right)$ : rol potencial de la exposición a la radiación ultravioleta. En: Efecto de los cambios globales en la diversidad. Programa CYTED Red 406RT0285.207-216 pp.

De los Ríos, P. 2008. A null model for explain crustacean zooplankton species associations in central and southern Patagonian inland waters. Anales Instituto Patagonia (Chile) 36: 25-33.

De los Ríos P. \& D. Soto 2009. Estudios limnológicos en lagos y lagunas del Parque Nacional Torres del Paine (51 ${ }^{\circ} \mathrm{S}$, Chile). Anales Instituto Patagonia (Chile) 37: 63-71

De los Ríos P, \& D. Soto 2007. Crustacean (Copepoda and Cladocera) zooplankton richness in Chilean Patagonian lakes. Crustaceana 80: 285-296.

Franca, F.G.R. \& A.F.B. Araújo 2007. Are there co-occurrence patterns that structure snake communities in Central Brazil?. Brazilian Journal of Biology 67: 33-40.

Frutos S.M. 1998. Densidad y diversidad del zooplancton en los ríos Salado y Negro, planicie del río Paraná- Argentina. Revista Brasileira de Biologia 58: 431-444.

Gotelli N.J. \& G.R. Graves 1996. Null models in Ecology. Smithsonian Institution Press, Washington, DC.

Gotelli, N.J. 2000. Null models of species co-occurrence patterns. Ecology, 81: 2606-2621.

Gotelli, N.J. 2001. Research frontiers in null model analysis. Global Ecology and Biogeography
10: 337-343.

Gotelli N.J. \& G.L Entsminger 2009. EcoSim: Null models software for ecology. Version 7. Acquired Intelligence Inc. \& Kesey-Bear. Jericho, VT 05465. http://garyentsminger. com/ecosim.htm.

Jaksic, F. 2001. Ecología de comunidades. Ediciones Pontificia Universidad Católica de Chile, Santiago. Marinone, M.C., S. Menu-Marque, D. Añón-Suárez, M.C. Diéguez, A. Pérez, P. De los Ríos, D. Soto \& H.E. Zagarese 2006. UV radiation as a potential driving force for zooplankton community structure in Patagonian lakes. Photochemistry and Photobiology, 82: 962-971.

Modenutti, B.E., E.G. Balseiro, C.P. Queimaliños, D.A. Suárez, M.C. Diéguez \& R.J. Albariño 1998. Structure and dynamics of food webs in Andean lakes. Lakes and Reservoirs, Research and its Management 3: 179-186.

Modenutti, B.E., C. Queimaliños, E. Balseiro \& M. Reissig 2003. Impacts of different zooplankton structures on the microbial food web of a South Andean oligotrophic lake. Acta Oecologica 24: 289-298.

Morris, D.P., H.E. Zagarese, C.E. Williamson, E.G. Balseiro, B.R. Hargreaves, B.E. Modenutti, R.E. Moeller \& C.P. Queimaliños 1995. The attenuation of solar ultraviolet radiation in lakes and the role of dissolved organic carbon. Limnology \& Oceanography 40: 1381-1391.

Pascual, M.A., V. Cussac, B. Dyer, D. Soto, P. Vigliano, S. Ortubay \& P. Macchi 2007. Freshwater fishes of Patagonia in the 21st Century after hundred years of human settlement, species introduction and environmental change. Aquatic Ecosystem Health and Management 10(2): 212-227.

Quirós, R. \& E. Drago 1999. The environmental state of Argentinean lakes: an overview. Lakes and Reservoirs, Research and its Management.4:55-64

Ramos-Jiliberto, R., J. Oyanadel, C. Vega-Retter \& F.S. Valdovinos 2009. Nested structure of plankton communities from Chilean freshwaters. Limnologica-Ecology and Management of Inland Waters, 39: 319-324.

Reissig, M., C. Trochine, C. Queimaliños, E. 
Balseiro \& B. Modenutti 2006. Impacts of fish introduction on planktonic food webs in lakes of the Patagonian Plateau. Biological Conservation, 132: 437-447.

Reissig, M., B. Modenutti, E. Balseiro \& C. Queimaliños 2004. The role of predaceous copepod Parabroteas sarsi in the pelagic food web of a large deep Andean lake. Hydrobiologia 524: 67-77.

Ribas C.R. \& J.H. Schoreder 2002. Are all ants mosaics caused by competition? Oecologia 131: 606-611.

Rodríguez-Fernández J.I., C.J. Barros de Carvalho \& M.O. Moura 2006. Estrutura de asembleias de Muscidae (Diptera) no Paraná: uma análise por modelos nulos. Revista Brasileira de Entomologia 50: 93-100.

Sanders N.J., G.M. Crutsinger, R.R. Majer \& J.H.C. Delabie 2007. An ant mosaic revisited: dominant ant species dissemble arboreal ant communities but co-occur randomly. Biotropica 39: 422-427.

Segurado P. \& D. Fiqueiredo 2007. Coexistence of two freshwater turtle species along a Mediterranean stream: the role of spatial and temporal heterogeneity. Acta Oecologica 32: 134-144.

Soto, D., I. Arismendi, C. Di Prinzio \& F. Jara 2007. Establishment of Chinook salmon (Oncorhynchus tshawytscha) in Pacific basins of southern South America and its potential ecosystem implications. Revista Chilena de Historia Natural, 80: 81-98.

Soto, D., I. Arismendi, J. González, J. Sanzana, F. Jara, C. Jara, E. Guzmán \& A. Lara 2006. Southern Chile, trout and salmon country: invasion patterns and threat for native species. Revista Chilena de Historia Natural,
79: 97-117.

Soto, D. 2002. Oligotrophic patterns in southern Chile lakes: the relevance of nutrients and mixing depth. Revista Chilena de Historia Natural,75: 377-393.

Soto, D., H. Campos, W. Steffen, O. Parra \& L. Zúñiga 1994. The Torres del Paine lake district (Chilean Patagonia): A case of potentially N-limited lakes and ponds. Archiv für Hydrobiologie 99:181-197.

Soto, D. \& P. De los Ríos 2006. Trophic status and conductivity as a regulators in daphnid dominance and zooplankton assemblages in lakes and ponds of Torres del Paine National Park. Biologia, Bratislava 61:541-546.

Soto, D. \& L. Zúñiga 1991. Zooplankton assemblages of Chilean temperate lakes: a comparison with North American counterparts. Revista Chilena de Historia Natural 64: 569-581.

Tiho, S. \& J. Johens 2007. Co-occurrence of earthworms in urban surroundings: a null models of community structure. European Journal of Soil Biology 43: 84-90.

Tondoh, J.E. 2006. Seasonal changes in earthworm diversity and community structure in central Côte d'Ivoire. European Journal of Soil Biology 42: 334-340.

Ulrich, W. 2004. Species co-occurrences and neutral models: reassessing J.M. Diamond's assembly rules. Oikos 107: 603-609.

Woelfl, S. 2007. The distribution of large mixotrophic ciliates (Stentor) in deep North Patagonian lakes (Chile): First results. Limnologica 37:28-36. 
